This is the accepted version of the following article: Borrego, C. et al. Explore and wait: a compositive routing-delivery scheme for relative profile-casting in opportunistic networks in Computer network, vol. 123 (Aug. 2017), p. 51-63.

Which has been published in final form at DOI 10.1016/.j.comnet.2017.05.007

(C) 2017. This manuscript version is made available under the CC-BY-NC-ND 4.0 license http://creativecommons.org/licenses/by-nc-nd/4.0/ 


\title{
Explore and Wait: A composite routing-delivery scheme for relative Profile-casting in Opportunistic Networks
}

\author{
Carlos Borrego ${ }^{\mathrm{a}, *}$, Adrián Sánchez-Carmona ${ }^{\mathrm{a}}$, Zhiyuan Li ${ }^{\mathrm{b}}$, Sergi Robles ${ }^{\mathrm{a}}$ \\ ${ }^{a}$ Department of Information and Communications Engineering \\ Autonomous University of Barcelona \\ Barcelona, Spain \\ ${ }^{b}$ Jiangsu High Technology Research Key Laboratory for Wireless Sensor Networks \\ Jiangsu, China
}

\begin{abstract}
In the context of Opportunistic Networking (OppNet), designing routing and delivery protocols is currently an open and active line of research. In some OppNet scenarios, destination addresses are not always known by sending applications. Profile-cast models solve this problem by allowing message destinations to be users or groups of users defined by their profiles. These profiles provide very effective ways of characterizing nodes in terms of node's attributes such as their profession, interests or typical whereabouts, for example. However, there are strong limitations in OppNet Profile-casting. There is no current way of representing special profiles defined by relative delivery functions such as best, maximum or over-the-average: nodes belong to these relative profiles taking into account not only attributes from the very same node but also relative to others from the same profile. In this article, we introduce Relcast, a Profile-cast model that allows messages to be sent to profiles defined in terms of relative delivery functions. Additionally, we present Explore and Wait, a composite routing-delivery scheme that uses optimal stopping theory-based delivery strategies to route Relcast messages. We show, using simulations that this routing-delivery scheme performs better than traditional approaches that use state-of-the-art routing-delivery primitives.
\end{abstract}

Keywords: OppNet, Bundle Protocol, Profile-cast, Optimization, DTN

\section{Introduction}

Opportunistic Networking (OppNet) [22] is a network paradigm where mobile nodes communicate with each other even if end-to-end connectivity among them never exists. In the context of OppNet, available destination addresses are not always known by the sending applications. Profile-based network models [13], where the destination of a message is not identified a by network/transport address, are very useful in OppNet. In this case, applications send messages to nodes belonging to a profile defined by one or more attributes rather than to specific addresses. For example, a user could send a message to another user belonging to a neighbour profile, defined as all users that live less than $1 \mathrm{~km}$ away from the current location.

However, there are strong limitations in OppNet Profilecasting. In particular, there is no current way of representing profiles defined by delivery functions such as best, maximum, over-the-average or $k$-best. We call these profiles relative profiles: nodes belong to these relative profiles

\footnotetext{
*Corresponding author

Email addresses: cborrego@deic.uab.cat (Carlos Borrego), adria.sanchez@deic.uab.cat (Adrián Sánchez-Carmona), lizhiyuan81@126.com (Zhiyuan Li), sergi.robles@uab.cat (Sergi Robles)
}

taking into account not only attributes from the very same node but also relative to others from the same profile. For instance, following the previous example, a user application might want to send a message to the user belonging to the neighbour profile that has visited a certain place more times than anyone else from this profile ${ }^{1}$.

Nonetheless, this relative delivery decision is a complex task to perform. The reason for this is that intermittently connected OppNet nodes do not have access to the state of the network with regard to the attributes that define these relative profiles. Broadcasting messages to query the state of these attributes can be inaccurate and slow because of the characteristics of the network. Instead, sending single messages to the network to explore the network and afterwards deliver the message will imply a new problem: the problem of deciding when to stop exploring the network.

In this article, we overcome these limitations by allowing messages to carry state variables to be able to implement complex delivery strategies. These strategies are based on optimal stopping theory, a statistical tool for analysing the problem of choosing an optimal time to take a particular

\footnotetext{
${ }^{1}$ In early versions of Foursquare social network, if a user had checked into a certain place more days than anyone else, he/she would become Mayor of that place.
} 
action. We introduce Relcast, a profile-based relative network model to allow messages to be sent to profiles using relative criteria. Additionally, we present, Explore and Wait, a composite routing-delivery scheme based on optimal stopping theory to optimise the delivery of Relcast messages.

Like other protocols as Spray and Wait [28], Explore and Wait operates in two phases. During the first phase, the Explore phase, the message studies the network and understands about the nodes attributes by being forwarded to as many nodes from this profile as possible. We propose using a profile-based probabilistic routing protocol to facilitate these contacts: messages are routed in terms of historical profile encounters. During the second phase, the Wait phase, the message has acquired enough knowledge about the network, and it is ready to be delivered. The message waits, while still being routed until the delivery strategy considers the message should be delivered to the forwarded node. The result is a general purpose networklayer scheme that provides OppNet applications with the possibility of implementing relative delivery strategies.

Finally, we present different useful applications and a wide set of simulations in two different scenarios: an urban and a rural scenario. We base our simulations on ActiveDTN [3, 2], an implementation of the Bundle Protocol that allows bundles to have an active routing and delivery behaviour. Active-DTN allows applications to send Relcast bundles by including within the bundle their delivery protocols as bundle extensions implemented with mobile code. The results of these simulations show that our proposal performs better than using traditional network primitives since Relcast allows new group communication behaviours that are capable of being addressed using optimal strategies.

The rest of the paper is organised as follows. Section 2 reviews the state of the art. Section 3 introduces the Relcast network model and the Explore and Wait protocol. In Section 4, the experimentation using simulations is described. Finally, in Section 5, results and the conclusions that are drawn are presented.

\section{Background}

In this section, we study the state of the art of three different OppNet issues directly related to the proposal of this study: Profile-casting, mobile code applied to OppNet and stateful routing. Additionally, we present a list of motivating scenarios that can take advantage of our proposal.

\subsection{Related Work}

\subsubsection{Profile-casting in OppNet}

Opportunistic Networking (OppNet) is a network paradigm where nodes suffer from intermittent contacts, long latencies, low delivery ratios and indeterminate mobility patterns. Messages in OppNet are routed from their source to their destination in an opportunistic way using intermediate nodes.

There have been some interesting proposals for profilebased delivery in OppNet. In [13], the authors present a novel delivery approach where messages are sent to a sub-group of users as defined by their profiles (e.g. interests, social affiliation, etc.). They study large data sets of user mobility profiles and present a case-study of mobility Profile-cast with a similarity-based forwarding protocol. The same authors have published a similar work [14] where a new behaviour-oriented communication paradigm in mobile networks named Profile-cast, motivated by tight user-network coupling in mobile societies, is presented. In this novel paradigm, the authors proposed that messages are sent to sender-specified target profiles, instead of machine IDs. They present a systematic framework for such services. Additionally, they present a communication protocol in mobile networks based on the stability of the user behavioral profile to discover the receivers implicitly, abreviated as CSI. In this protocol, messages are forwarded to a node if this node has a higher similarity to the target profile than the custodian of the message.

Identifying users according to their affiliation, as first presented by [15], can improve forwarding decisions. In [8], the authors propose an efficient social profile-based routing scheme. The efficiency of this proposed scheme has been confirmed by trace-driven simulation, which also reflects the efficacy of exploring social features in OppNet. In [21], the authors present Peoplerank, a forwarding approach similar to PageRank where nodes are ranked using weighted social information. In a similar way, the authors of [34] introduce Predict and Relay, a routing algorithm based on the foreseeability and semi-deterministic node's movement models.

Profile-cast seems to be a very interesting network model in OppNet. However, traditional delivery strategies are limited to node attributes. When facing more complex delivery strategies like, for example, relative-based ones, such as sending messages to the best node from a given profile, these proposals fail to give good general and dynamic network-layer solutions. As a consequence of this, some applications are not possible in OppNet.

\subsubsection{Stateful routing in OppNet}

Several proposals in OppNet have been presented to include message state information in OppNet messages to improve OppNet issues such as message routing. For example, in [28], the popular Spray and Wait routing protocol sprays a number of copies into the network, and then waits until one of these nodes meets the destination. The number of times a message can be replicated is proposed to be included in the message, and it is decreased every time the message is sprayed. This is a very good example of message state. Unfortunately, these message states are not able to be implemented in the Bundle Protocol: there is no current proposal for including such state in the Bundle Protocol messages. 
Mobile code along with message state has proven to be a good combination in a variety of settings. In [3] software code and message state are presented to improve OppNet performance. The authors provide a solution that consists in extending the messages being communicated by incorporating software code for forwarding, delivery, lifetime control, and prioritisation purposes. The proposal stems from the idea of moving the routing and the delivery algorithms from the router to the message.

Finally, very recently, proposals like [17] have started to apply to OppNet optimal stopping theories [5, 23], statistical solutions for the problem of choosing a moment to make a particular decision to maximise a certain reward. This statistical approach has been applied to network issues such as routing or node searching. However, although these proposals need message state variables to be able to make optimal decisions on every contacted node, the authors of these studies do not provide information about it.

\subsection{Motivating Scenarios}

In this section, we discuss some motivating scenarios where traditional Profile-cast models fail to give efficient solutions.

\subsubsection{Urban $O p p N e t$}

As explained in studies like [29], Internet service providers (ISPs) are using package inspection techniques to read and store user's messages and personal data. The authors of this study explain that these technologies are progressively gaining legal legitimacy. Additionally, cloud services can ban users when connecting using Tor-like anonymous services ${ }^{2}$.

As a consequence of this, OppNet has become a network paradigm that can be used as an alternative to traditional Internet infrastructure-based networks in order to preserve privacy issues such as message source, user's location, travel habits, interests, wealth, social network and many others.

However, in some OppNet scenarios like urban OppNet scenarios [27], destination addresses may be unknown to the senders. Instead, profile-casting models can be very useful to define message destinations. However, there are current limitations on OppNet Profile-casting. These limitations include, for instance, the cases when messages have an implicit cost when being delivered. An example of this can be an advertisement application where users are willing to receive custom designed advertisement messages in terms of their interests. In this case, sending applications may want to optimise the destination users in terms of one or more profile attributes. An advertisement message will be sent asynchronously to a network formed by user's mobile devices to users that satisfy best a given criterion.

\footnotetext{
${ }^{2} \mathrm{~A}$ list of services blocking TOR: trac.torproject.org/projects/tor/wiki/org/doc/ListOfServicesBlockingTor
}

Users may reveal their interests to these applications preserving their privacy by not revealing their identities to Internet service providers. Current Profile-cast models are not able to implement these delivery optimisations.

\subsubsection{Rural OppNet}

In remote rural areas, traditional network infrastructures are not always fully deployed [35]. In these rural scenarios, OppNet can be a good network solution for communication purposes, as seen in studies like [24], where a rural podcast distribution intermittently connected network in the rural area of Cajamarca, Peru is presented. Communication among the different farmers may be very useful in order to allow, for example, agricultural knowledge networks or goods exchange networks to be created. Farmers can form an OppNet network using already deployed mobile devices and opportunistic contacts.

However, current Profile-casting models fail to provide efficient solutions to allow farmers to send messages to those farmers that maximise a certain criterion like knowledge of a particular product, a provision of a good, or a good exchange ratio, for example. In these cases, messages could be sent to farmers that satisfy best this type of criteria to perform orders or goods reservations in order to make future exchanges when they physically see each other in a local market, for example.

\subsubsection{Influencer delivery in Mobile Ad Hoc Social Net- works}

Very recently, in the context of OppNet, research is directed towards a new network paradigm that evolves from the traditional node-to-node scheme to a more personcentric one [6]. The research community has realised that is possible to discover important characteristic properties of social networks from different sources such as sets of real-world human contact traces. The result of these research studies are many social-based routing protocols $[16,7,31,36,33]$ that present different graph-based network-analysis metrics such as network centrality, social similarity, data/interest similarity, social relationship and community metrics to enhance message delivery performance.

When OppNet is seen from a social perspective, new network roles come into view. For example, OppNet nodes can be profiled in terms of how socially connected they are. Finding strongly connected nodes in OppNet is far from being easy to achieve due to its dynamical change of topology and the lack of a global view of the network. Current Profile-cast OppNet models fail to provide solutions for the delivery problem that allow, in an optimal way, to deliver messages to special profiles defined by nodes which are highly socially connected. These nodes are generally characterised by having interesting attributes such as high reputation, trustfulness and credibility.

This OppNet network role opens new avenues for social media opportunistic applications where users could be contacted in terms of how social they are. 


\subsubsection{Device-to-Device Opportunistic Networks}

Very lately, a new opportunistic network approach named Opportunistic device-to-device (D2D) [10, 4] communication has caught the attention of the research community. In opportunistic D2D, different devices communicate with each other in an autonomous way without having any centralised infrastructure. The purpose of these connections is to gather, share, and forward information in a multi-hop manner. As explained in [1], this opportunistic D2D network paradigm can play a very important role in the development of the Internet of Things (IoT).

The number of this type of connected devices is growing at a breathtaking pace. As explained in [14], the Profilecasting paradigm enables many behaviour-oriented services efficiently. When having so many devices connected together, the intelligence of the IoT applications is not only located in what to send but also to whom. For these purposes, these Profile-casting models may be very useful to categorise these different types of device behaviour. However, current Profile-casting models are limited when it comes to defining device profiles in terms of characteristics that take into account not only the very same device but also others from the same type. As a consequence of this, Opportunistic D2D networking does not have optimal ways of allowing applications to select as the destination of their messages very useful relative profiles defined by relative delivery functions such as best or maximum.

Even though there has been considerable ongoing work on Profile-casting for OppNet, there is yet some open lines of research. We explain in this study that current Profilecasting solutions for OppNet are limited when it comes to defining node profiles taking into account not only characteristics from the very same node but also relative to others.

\section{Explore and Wait: a Composite Routing- Delivery Relcast Scheme}

In this section, we describe Relcast, a relative Profilecast model for OppNet and Explore and Wait, a novel composite routing-delivery scheme to implement, from the network layer perspective, Relcast strategies to improve Profile-cast communications. In order to improve the readability of this section, in Table 1, a summary of the terms explained in this section is presented.

\subsection{Relcast: Motivation and Definition}

Traditional Profile-cast schemes, such as the ones presented in Section 2, deliver messages to a group of nodes in a network identified by their profiles (see Figure 1). These profiles are very useful when available destination addresses are not known by sending applications, and provide very effective ways of characterising nodes in terms

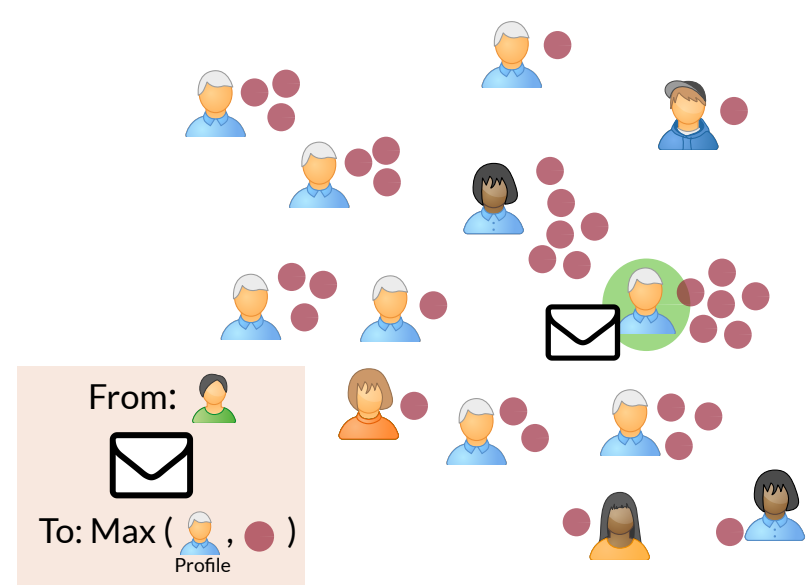

Figure 1: Different nodes in a network belonging to different profiles with different values for a certain attribute (dark circle in the figure). As an example of relative delivery, a Relcast message is sent to a single node that belongs to the white hair profile that maximises the value of this attribute.

of node's attributes such as their professions, interests or historical whereabouts, for example.

However, node profile characterising is a complex issue, specially in scenarios where the values of these attributes are unknown to the sender application. In these cases, it may be very useful to be able to send messages not to a profile itself, but to a subset of the nodes belonging to this profile in terms of relative criteria. We call these Profilecast model Relcast.

In order to formally define a relative delivery function, we denote by $N$ as the set of all the $n$ nodes in an OppNet network, $N=\left\{\right.$ node $_{1}$, node $_{2}, \cdots$, node $\left._{n}\right\}$. We define $\mathcal{P}(R)$ as:

$$
\mathcal{P}(R)=\left\{R_{1}, R_{2}, \cdots, R_{r} / R_{j} \subseteq R \backslash \emptyset\right\} .
$$

Let $\mathcal{P}(N)$ be the set of all the non-empty subsets of $N$ that represent the set of all the possible lists of nodes belonging to any given profile. Given a profile $p$, we define $P_{p} \in \mathcal{P}(N)$ as the set of nodes that belong to this profile.

$$
\begin{gathered}
P_{p}=\left\{\text { node }_{1}, \text { node }_{2}, \cdots, \text { node }_{t} /\right. \\
\text { node } \left.\left._{j} \in N \quad \& \text { belongs }\left(\text { node }_{j}, p\right) \quad \forall j \in[1, t]\right)\right\} .
\end{gathered}
$$

Given a profile $p$, a $P_{p} \in \mathcal{P}(N)$ and a node attribute ${ }^{3} a$, we now can define a relative delivery function as:

$$
\text { relf: } P_{p} \text { х } a \rightarrow \mathcal{P}\left(P_{p}\right) .
$$

We define a Relcast message as a message sent to a relative profile, a special OppNet profile that characterises a node that belongs to the list of nodes in a network that satisfy a certain relative delivery function. Additionally, we define target profile as a profile that characterises an OppNet node that belongs to the list of all the possible delivery candidate nodes for a certain relative delivery function.

\footnotetext{
${ }^{3}$ We use attribute in singular, but more than one attribute can be considered as a single compound attribute.
} 


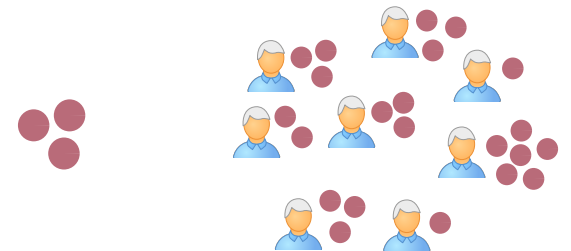

(a) Node attribute (b) Target profile.

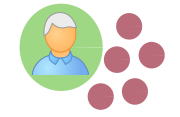

(c) Relative profile

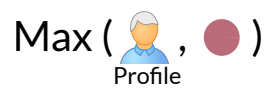

(d) Relative delivery function

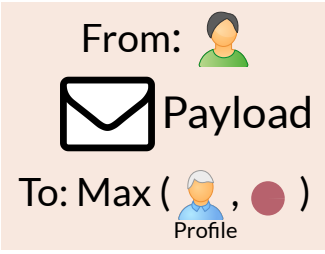

(e) Relcast message

Figure 2: Different terms introduced in this section using as an example Figure 1 and depicted separately: (a) a node attribute, (b) a target profile representing nodes with white hair, (c) a relative profile that represents a profile defined by the best member belonging to the target profile that maximises the value of the node attribute, (d) a maximum relative delivery function and (e) a Relcast message.

Given a target profile $c$, a relative delivery function relf, a source node source and a payload payload, we formally define a Relcast message $m$ as $m$ (source, payload, $p$, relf).

It is important to understand that the the relative delivery function takes as an argument $P_{p}$, the list of all the nodes belonging to a certain profile. This is why message delivery in Relcast is a complex task in OppNet: relative delivery decisions take into account the value of a certain attribute for every node belonging to a target profile.

Following the previous example, the target profile would be the neighbour profile and the relative profile could be the user that has visited a certain place more times than anyone else. A Relcast message, then, is defined as a message sent by a source node, with a certain payload to the relative profile that represents the user that has visited a certain place more times than anyone else.

In Figure 2, the terms introduced in this section using as an example the one of Figure 1 are depicted separately.

\subsection{Explore and Wait scheme}

In the previous sections, we have introduced Relcast messages as messages sent to a relative profile, a special OppNet profile that characterises a node that belongs to the list of nodes in a network that satisfy a certain relative delivery function. In this section, we explain how to optimally deliver these messages.

In traditional networks, such as TCP/IP, it is not directly possible to send a message to a node characterised by a relative profile. However the same service can be obtained by following a three-phase strategy using classical network primitives such as Unicast and Broadcast. Firstly, a Broadcast attribute query message may be sent to all of the members belonging to the target profile. Then (Step 2 in the same figure), once received the response from the target profile members and these responses are ordered in terms of the studied attribute, $k$ nodes are selected using the relative delivery function, such as best, over-the-average, or $k$-best, for example. Finally (Step 3 in the same figure), $k$ Unicast messages are sent to this selection of nodes. We obtain by following this scheme a Profile-cast delivery that selects one or more nodes from a given target profile that satisfies a given relative criteria.

However, in OppNet, this three-phase strategy scheme does not work efficiently: Broadcast query messages (Step
1) or their responses (Step 2) may not be delivered, because of the idiosyncrasy of the intermittently connected network. Consequently, the selection of the nodes may be inaccurate. Even if properly selected, the Unicast messages (Step 3) may not arrive either to their destination for the very same reason.

This is why we propose a different scheme for Relcasting that can be efficiently and optimally applied to OppNet. Our proposal consists of a two-phase strategy implemented by a single Relcast message, as depicted in Figure 3. Firstly, during the Explore phase, the Relcast message is forwarded from one node to another. While in this phase, the Relcast message understands the state of the network as to the studied attribute. This is done by retrieving the values for the studied attribute from every node the message is forwarded to. Secondly, the Relcast message switches to the next phase: the Wait phase. While in this phase, the Relcast message has already gained the knowledge about the network, and it is capable of deciding when and how to perform the delivery action for every node it is forwarded to. The Relcast message waits during this phase, while being routed, until it can find a node or a group of nodes that meet certain requirements to complete the delivery action.

We call this network scheme Explore and Wait: a twophase strategy that combines routing actions and delivery decisions.

\subsection{Explore Phase: Exploring the Network}

As depicted in Figure 3, different nodes belonging to a certain target profile may have different values for a given attribute. Applications, at sending time, may experience the "millionaire at the billionaire party" problem" ${ }^{4}$ it is difficult to know which are good/high or bad/low values for a node attribute relative to other nodes from the same target profile. This is why we propose to, first, allow the Relcast messages to understand the state of the network according to this studied attribute.

During the Explore phase, Relcast messages are not delivered. Instead, they are just routed from one node to

\footnotetext{
${ }^{4} \mathrm{~A}$ hypothetical situation where a millionaire arrives at a party where everybody is billionaire and boastfully displays its wealth.
} 


\begin{tabular}{|c|c|}
\hline Name & Description \\
\hline Target profile & $\begin{array}{l}\text { Group of nodes in an OppNet defined in terms of one or more attributes. } \\
\text { Example: neighbour profile attribute }\end{array}$ \\
\hline Attribute & $\begin{array}{l}\text { Quality of a node/user that defines a profile. Example: User's distance } \\
\text { from home. }\end{array}$ \\
\hline Relative delivery function $(\mathrm{relf})$ & $\begin{array}{l}\text { Delivery function where nodes are selected taking into account } \\
\text { not only attributes from the very same node, but also relative to others } \\
\text { from the same profile. Example: best and second best members of a profile } \\
\text { in terms of an attribute. }\end{array}$ \\
\hline Relative profile & $\begin{array}{l}\text { Subset of a target profile that satisfies a relative delivery function. } \\
\text { Example: best person of the neighbour profile in terms of a certain attribute }\end{array}$ \\
\hline Relcast message & $\begin{array}{l}\text { A Profile-cast message sent to a relative profile in terms of a } \\
\text { relative delivery function }\end{array}$ \\
\hline Optimal Phase Transition Value $(\sigma)$ & $\begin{array}{l}\text { Optimal value of the number of forwarded nodes belonging to a certain profil } \\
\text { that maximises the probability of delivery success. }\end{array}$ \\
\hline Message Profile Scope $\left(P_{s}\right)$ & $\begin{array}{l}\text { Estimation of the number of potential nodes belonging to a certain profile } \\
\text { the message can be forwarded to for a given period of time }\end{array}$ \\
\hline Measured Inter-Forward Time $\left(I F T_{m}\right)$ & $\begin{array}{l}\text { Measured time between the end of the time the message previously } \\
\text { contacted a node and the beginning of a new contact. }\end{array}$ \\
\hline Smooth Inter-Forward Time (SIFT) & Smooth average of the Measured Inter-Forward Time \\
\hline Measured Profile Membership $\left(P M_{m}\right)$ & Measured profile belonging \\
\hline Smooth Profile Membership $(S P M)$ & $\begin{array}{l}\text { Smooth average of the list of the Measured Profile Membership of the list of } \\
\text { nodes a message has been forwarded to }\end{array}$ \\
\hline$\alpha$ & $\begin{array}{l}\text { Constant to give different weights among historical values or new measured } \\
\text { values when calculating SIFT. }\end{array}$ \\
\hline$\omega$ & $\begin{array}{l}\text { Constant to give different weights among historical values or new measured } \\
\text { values when calculating SPM. }\end{array}$ \\
\hline Profile Forwarding Metric $\mathrm{P}(\mathrm{p}, \mathrm{n})$ & Probability of a given node (n) encountering a certain profile (p). \\
\hline$\gamma$ & \\
\hline & Profile-based probabilistic routing transitivity weight constant. \\
\hline
\end{tabular}

Table 1: Summary of the terms presented in this section.

another. Understanding the network according to the attribute is done by querying the studied attribute value when a Relcast message is being routed to a node in order to build a statistic about it. This statistic is carried by the Relcast message during the Explore phase, and it is updated by retrieving new values from the different nodes the Relcast message is forwarded to.

Feeding the Relcast message Explore phase statistic with new node attributes can be made in very different ways. The Relcast message can use simple statistic operations such as maximum, minimum or average or some more complex ones such as cumulative, or weighted averages. The richer this statistic is, the more representative from the studied attribute would be but likewise, it would be more expensive in terms of network, storage and computation resources.

The node's attribute values may be seen as a private information a node would not want to share. We would like to note that there are many mechanisms that can help to improve security in this type of delivery schemes. For example, we propose to use identity-based access control techniques, as the authors of this paper have already employed in studies like [25]. By using these identity-based access control techniques, only certain delivery and software codes identified by their own hashes, are allowed to be executed in a node. These techniques may help to prevent delivery software codes like the one presented in Algorithm 1 to perform malicious practices such as retrieving attribute values for the sole purpose of revealing them.

\subsubsection{A Probabilistic Profile-based Routing Protocol}

In OppNet, for a given message, a routing protocol chooses, among the different contacted nodes, which subset of them the message should be forwarded to. Routing protocols like the probabilistic routing protocol PRoPHET [19] take into account the fact that some DTN nodes have predictable movement models such that if a node has frequently contacted another node in the past, it is likely that it will be contacted again in the future. We formulate the hypothesis that will be simulated and analysed in Section 4: in the very same way the authors of PRoPHET suggest, if a node has contacted another node from a certain profile in the past, it is also likely that it will contact the same node or another from this profile again in the future.

Our objective is to make the Explore phase as richer as possible: the more visited nodes from a target profile, the 


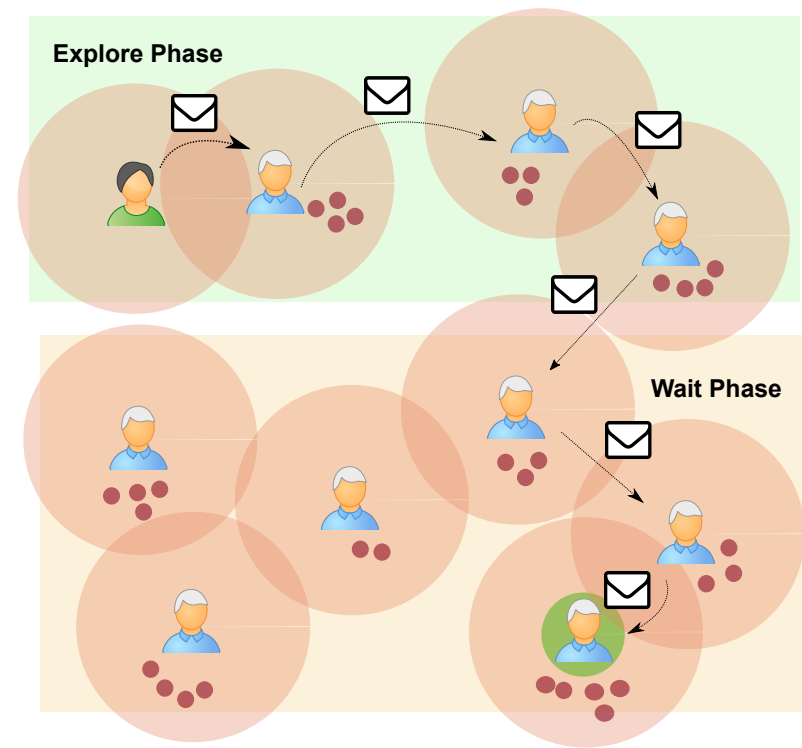

Figure 3: Explore and Wait scheme: a single Relcast message is sent to the best node in terms of a certain attribute (dark circles). While in the Explore phase, this Relcast message is being forwarded to three nodes. During this first phase, the Relcast message gains information about the network as to the studied attribute. After the third node is being forwarded to, the Relcast message switches to the Wait phase. Finally, the message is forwarded to the first node that satisfies the delivery requirements taking into account the gained information from the previous phase.

better will be the delivery decision.

As in PRoPHET, we define a routing metric, the profile forwarding metric, $P($ profile, node $) \in[0,1]$, that represents the probability of a given node encountering a node from a certain profile $p$. Whenever a node $A$ encounters another node from a given profile $p$, the profile forwarding metric is updated using the following expression, where $P_{\text {init }}$ is an initialization constant:

$$
P(p, A)_{\text {new }}=P(p, A)_{\text {old }}+\left(1-P(p, A)_{\text {old }}\right) \cdot P_{\text {init }} .
$$

The predictabilities for all profiles are aged using the following ageing expression, being $\gamma \in[0,1]$ an ageing constant and $k$ is the number of time units that have passed since the last ageing update.

$$
P(p, A)_{\text {new }}=P(p, A)_{\text {old }} \cdot \gamma^{k} .
$$

Finally, the profile delivery predictability, as in PRoPHET, is updated in a transitive way. In the following equation, we show how this transitivity modifies the profile forwarding metric. We use $\delta \in[0,1]$, a constant to specify the weight of how this transitivity should affect the profile forwarding metric. When a node $A$ that belongs to a profile $p_{a}$ contacts node $B$ that belongs to a profile $p_{b}$ and that has a profile forwarding metric for profile $p_{c}$ with value $P\left(p_{c}, B\right)$, then:

$$
\begin{aligned}
P\left(p_{c}, A\right)_{\text {new }}= & P\left(p_{c}, A\right)_{\text {old }}+ \\
& \left(1-P\left(p_{c}, A\right)\right) \cdot P\left(p_{b}, A\right) \cdot P\left(p_{b}, B\right) \cdot \delta .
\end{aligned}
$$

Messages are forwarded in terms of these delivery predictabilities. If a node A has in custody a Relcast message with destination a relative profile that considers the following general relative delivery function:

$$
\text { relf: } P_{p} \text { х } a \rightarrow \mathcal{P}\left(P_{p}\right) .
$$

then, A will forward this message to a node $B$ if $P(p, A)<P(p, B)$. Note that this probabilistic routing protocol, as opposed to PRoPHET [19], keeps a single copy of the Relcast message.

The node's profile forwarding metrics may be seen as a private information a node would not want to share. Security is out of the scope of this article, however, we would like to note that these profile forwarding metrics may be kept private while implementing our probabilistic profileaware routing protocol by using homomorphic encryption. This cryptographic technique allows nodes to carry out routing arithmetical operations on their encrypted profile forwarding metrics without the need of decrypting them first. As a consequence of this, the comparison of the node's profile forwarding metrics can be computed without revealing the actual values. The authors of this study have already used this technique in studies like [26] to implement a private georouting protocol that preserves nodes' privacy while taking routing decisions. In this study a proof of concept is presented and shows that homomorphic encryption can be used with low powerful devices.

\subsection{On State Change: from Explore Phase to Wait Phase using Optimal Stopping}

The Explore and Wait scheme is a composite routing and delivery strategy to optimise the delivery of Relcast messages. The longer the Explore phase is, the richer the message statistic about the state of the network according to the studied attribute will be. However, since during the Explore phase messages are not delivered, an excessively long Explore phase may prevent many messages from being considered for delivery on certain nodes belonging to the target profile. This makes the phase transition a very complex decision to be made: an early phase transition implies poor knowledge of the network while a late phase transition implies missing good candidates for message delivery.

In statistics, the optimal stopping theory [5] deals with this problem: choosing a moment to take a particular action, in order to maximise an expected reward or minimise an expected cost. In OppNet, the problem is even more complicated since it is difficult to know in advance the number of nodes from the target profile a message will be forwarded to. However, there is an analytical solution to this statistical problem: there is an optimal value that maximises the probability of delivery success. Nevertheless, this optimal Phase Transition Value $(\sigma)$ cannot be always found and depends on the relative delivery function. Following, we analyse in detail this problem for a concrete relative delivery function, the maximum relative delivery 


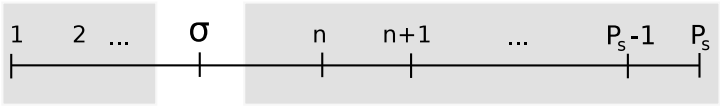

Optimal phase transition

Figure 4: Number of nodes belonging to the target profile a Relcast message has been forwarded to before switching to the Wait phase. Early phase transition implies poor knowledge of the network while late phase transition implies missing good candidates for Relcast message delivery. The optimal Phase Transition Value is represented by $\sigma$.

function: the problem of delivering a Relcast message to a node from a target profile that maximises the value of the studied attribute. We consider for this delivery problem that the bigger this value is, the better. Additionally, the solutions to the optimal Phase Transition Value for other relative delivery functions are provided.

\subsubsection{Example of an Optimal Phase Transition Value calculation: the maximum relative delivery function}

In Figure 4, we represent $n$, the number of nodes belonging to the target profile a Relcast message has been forwarded to before switching to the Wait phase. We consider the optimal Phase Transition Value $(\sigma)$ as the optimal last node not to be considered for delivery, that is, the last node in the Explore phase. We define node* as the best node to deliver the Relcast message according to the maximum relative delivery function. Let node node* to be contacted at $n+1$. We define $P_{s}$ (Message Profile Scope) as the number of nodes belonging to a certain target profile a message can be forwarded to in given period of time ${ }^{5}$. Then, a Relcast message will be delivered to node* if the following conditions are met:

- $n \geq \sigma$

- The maximum attribute value found in $[1, n]$ is the same as in $[1, \sigma]$.

Since the probability of optimal delivery is $1 / P_{s}$, the probability of this last condition happening is $\frac{\sigma}{n} \cdot \frac{1}{P_{s}}$. Adding all possible $n \geq \sigma$ we obtain:

$$
P(\sigma)=\frac{1}{P_{s}} \cdot\left[\frac{\sigma}{\sigma}+\frac{\sigma}{\sigma+1}+\frac{\sigma}{\sigma+2}+\cdots+\frac{\sigma}{P_{s}-1}\right],
$$

which is equivalent to:

$$
P(\sigma)=\frac{\sigma}{P_{s}} \cdot \sum_{n=\sigma}^{P_{s}-1} \cdot \frac{1}{n} .
$$

Hence:

\footnotetext{
${ }^{5}$ In OppNet, this Message Profile Scope is very difficult to predict at sending time. We will explain in Section 3.6 how to make an estimation of this variable.
}

$$
P(\sigma)=\lim _{P_{s} \rightarrow \infty} \frac{\sigma}{P_{s}} \sum_{n=\sigma}^{P_{s}-1} \frac{P_{s}}{n} \frac{1}{P_{s}} .
$$

In the work by Ferguson et al. [11], a review of different Optimal Stopping theory problems, the author express this limit expression as an approximation to an integral:

$$
P(\sigma)=x \int_{x}^{1} \frac{1}{t} \cdot d t=-x \cdot \ln x
$$

To obtain the optimal value we set the derivative to zero:

$$
P^{\prime}(\sigma)=-\ln x-1=0, x=1 / e .
$$

The expression $\sigma / P_{s}$ is optimal at $1 / e$. So for $P_{s} \gg 1$ the optimal $\sigma$ is $P_{s} / e$. This means that for the maximum relative delivery function, the optimal strategy is stop $e x$ ploring elements after $\sigma=P_{s} / e$ nodes contacted.

Other relative delivery functions have different optimal $\sigma$. For example, the kth-maximum relative delivery function, the problem of delivering a message, not to node that has the maximum value for the studied attribute but to the kth one, also has an optimal stopping solution. For instance, for $k=2$, this problem is similar to the Postdoc problem ${ }^{6}$, as presented and solved in [32], the value $P_{s} / 2$ is obtained as its optimal $\sigma$. In the context of the $k$ maximum relative delivery function, the problem of delivering a message to the $k$ elements from a target profile that maximises an attribute value, in [12], a review of different optimal stopping problems and its extensions, a solution to this problem is analytically described and considers, for $k=2$, an optimal $\sigma=P_{s} / 4.3649$.

Consequently, for a given relative delivery function, we propose to switch from the Explore phase to Wait phase when the Relcast message has been forwarded to the $o p$ timal Phase Transition Value $(\sigma)$ nodes from the target profile. This $\sigma$, as seen in the different examples exposed in this section, is a value calculated in terms of the estimation of the Message Profile Scope $\left(P_{s}\right)$. This estimation of the nodes a Relcast message can be forwarded to is not a trivial matter, and it will be deeply analysed in Section 3.6 .

\subsection{Wait Phase: Message Delivering}

During the Wait phase a statistic accurate enough to understand the state of the network according to the studied attribute has been created and it is stored within the Relcast message. From this moment, the Relcast message delivery action can be performed. In this phase, the routing protocol behaves in a very similar way as in the Wait phase in Spray and Wait. In Explore and Wait during the Wait phase, the Relcast message waits until it finds one or more nodes satisfy a relative delivery function in terms of the information learnt from the Explore phase according to the built statistic.

\footnotetext{
${ }^{6}$ The problem of delivering a message not the maximum but to the second maximum from a pool of $n$ candidates.
} 
The Wait phase is highly related to the chosen relative delivery function. Each relative delivery function is achieved by following a different delivery strategy. For example, in the case of the maximum relative delivery function, the optimal strategy is a well-known statistical problem [12]. Its solution is a strategy that rejects the first $P_{s} / e$ nodes (Explore phase) and then waits to deliver the Relcast message to the first contacted node that is better than all of the previous ones in terms of the target profile attribute (Wait phase).

Other relative delivery functions, such as over-theaverage or $k$-maximum imply similar strategies that can be implemented as well from a statistical perspective, as explained in [12].

\subsection{Message Profile Scope}

We study in this section how to estimate the Message Profile Scope $\left(P_{s}\right)$, the number of potential nodes belonging to a certain target profile a Relcast message can be forwarded to for a given period of time. This number, in the majority of OppNet scenarios, cannot be known in advance. It depends on the node's movement models, which in many OppNet scenarios can be very unpredictable. We propose in order to estimate the Message Profile Scope, to keep within every Relcast message two statistics. The first statistic is a statistic of the frequency of forwarded nodes. This statistic of the message Inter-Forward Time is an average of the time of two successive forwarding actions. The second statistic is a statistic of the percentage of the nodes a message has been forwarded to that belong to the target profile.

The algorithm to build these statistics must be an adaptive one, very light in terms of storage space and that takes into account both historical information from the past frequency measured values and new information from new ones. As Transmission Control Protocol (TCP) does for the estimation of its round trip time (RTT), we propose to use an Exponentially Weighted Moving Average [20], an adaptive statistical algorithm that applies weighting factors which decrease exponentially.

In order to build the first statistic, that we will call it the Smooth Inter-Forward Time (SIFT), when a Relcast message is forwarded to another node, the measured Inter-Forward Time $\left(I F T_{m}\right)$ defined as the following is calculated:

$$
I F T_{m}=\text { time.now }()-\text { lastfwdtime, }
$$

where time.now is the current time and lastfwdtime is the time of the Relcast message was last forwarded:

The SIFT will be updated following the expression:

$$
\text { SIFTnew }=\alpha \cdot S I F T_{\text {old }}+(1-\alpha) \cdot \text { IFT }_{m}, \alpha \in[0,1],
$$

where SFIt old is the historical SIFT, $\alpha$ a way of giving different weights to historical values and new measured values and $I F T_{m}$ the last Inter-Forward time measured.
In order to build the second statistic, that we will call it Smooth Profile Membership (SPM), every time a Relcast message is forwarded to a node, the Measured Profile Membership $\left(P M_{m}\right)$ is calculated as:

$$
P M_{m}= \begin{cases}1, & \text { if belongs }(\text { node }, \text { profile }), \\ 0, & \text { otherwise. }\end{cases}
$$

The Smooth Profile Membership is calculated by using the following expression:

$$
S P M n e w=\omega \cdot P M_{o l d}+(1-\omega) \cdot P M_{m}, \omega \in[0,1] .
$$

Once these statistics are updated, the Message Profile Scope $\left(P_{s}\right)$ can be calculated as the estimation of the number of potential nodes that a Relcast message can be forwarded to from a certain target profile in a period of time (t) in terms of the calculated SIFT and SPM. This way of estimating the $P_{s}(\mathrm{t})$ is:

$$
P_{s}(t)=t \cdot S P M / S I F T
$$

$\overline{\text { Algorithm } 1 \text { Example of a delivery software code for the }}$ maximum delivery function.

Ensure: $p$ is the target profile.

Ensure: forwarded is a message state variable with the number of nodes this message has been forwarded to.

Ensure: lastfwdtime is a message state variable with the time of the last forwarded node.

Ensure: $S I F T$ and $S P M$ are message state variables with the statistic of the Smooth Inter-Forward Time and the Smooth Profile Membership respectively.

Ensure: $t$ is the time limit for message delivery set to 3 hours (10.800 seconds).

Ensure: $p_{\text {value }}$ is the value of the studied attribute.

Ensure: $\max$ is a message state variable with the maximum $p_{\text {value }}$ found.

1: procedure DELIVER

$$
\mathrm{t}=10800
$$

forwarded ++

if belongs(localhost,p) then

else

$$
P M_{m}=1
$$

$P M_{m}=0$

end if

$S P M_{m}=\omega \cdot S P M+(1-\omega) \cdot P M_{m}$

$S I F T_{m}=$ time.now () - lastfwdtime

$S I F T=\alpha \cdot S I F T+(1-\alpha) \cdot S I F T_{m}$

$P_{s}=t \cdot S P M / S I F T$

if forwarded $>P_{s} / e$ and $p_{\text {value }}>\max$ then

end if deliver(msg)

if $p_{\text {value }}>\max$ then

end if

end procedure 


\section{Experimentation}

The Relcast Profile-cast model allows new applications to emerge in very different OppNet scenarios. In this section, we suggest, as an example, two different applications based on two of the different scenarios introduced in Section 2.2. Additionally, we present a series of simulations of these applications from two different OppNet scenarios: an urban and a rural scenario. We have chosen two scenarios to see how different scenarios may adapt and benefit from our proposal. Finally, we study if our proposal performs better than other based on state of the art network primitives such as Broadcast or Unicast.

\subsection{Applications and Scenarios}

The first scenario analysed is an urban scenario. As introduced in Section 2.2.1, we model a travel-like-a-local application where a tourist visiting a city would like to ask locals for restaurant recommendations or things to visit in town but without using traditional connected networks in order to preserve their privacy. In this scenario, we simulate how messages are sent asynchronously using a OppNet formed by the user's mobile devices to a local person that satisfies best a given criterion, such as affinity or reputation.

The second scenario analysed is a rural scenario similar to the one presented in [24], where a podcast distribution application is deployed in the remote rural area of Cajamarca, Peru. In this type of areas, network coverage does not cover all the rural area. However, communication among the different farmers may be very useful in order to allow agricultural knowledge networks or goods exchange networks, for example, to be created.

In this scenario, we simulate how farmers form an OppNet network using already deployed mobile devices and opportunistic contacts. We study the behaviour of messages sent to farmers that maximise a certain criterion like knowledge of a particular product, a provision of a good, or an exchange good ratio, for example. The destination of these messages are farmers identified by the profile that satisfy best this type of criteria with the purpose of performing orders or good reservations in order to make future exchanges, when they physically see each other in a local market, for example.

\subsubsection{Simulation Objectives}

The goal of these simulations is to understand the performance of the Explore and Wait scheme when several Relcast messages are sent to nodes belonging to a relative profile defined by the maximum relative delivery function in a given time period. Consequently, the bigger the value retrieved from the node chosen for delivery, the better. We have chosen a period of time of 3 hours which is big enough to allow messages in both scenarios to be forwarded to a representative number of nodes belonging to the target profile. The studied node attribute is simulated using a value that follows a random distribution between 0 and 1 .
In order to solve the problem of sending a Relcast message to a node from a given target profile that maximises a certain attribute, we have followed in the simulations presented in this section for both scenarios the strategy defined in Sections 3.3, 3.4 and 3.5. We have programmed the simulator to allow the Relcast messages when being created to remain in the Wait phase and retrieving the studied attribute from every node it the message is forwarded to. The statistic the Relcast messages keep for the studied attribute, as introduced in Section 3.3, is the maximum value. Messages keep an estimation of their Message Profile Scope $\left(P_{s}\right)$, that is the number of nodes to be forwarded $\left(P_{s}\right)$, as described in Section 3.6. As introduced in Section 3.6, these simulations will calculate the optimal Phase Transition Value $(\sigma)$, the optimal value that maximises the probability of delivery success depending on the delivery function. We follow in these simulations the state change strategy introduced in Section 3.4: when the number of nodes the Relcast message has been forwarded to is bigger than $\sigma^{7}$, messages switch to the Wait phase. Additionally, as explained in Section 3.5, during these simulations, messages were delivered to the first node that was better than all of the previous ones in terms of the target profile attribute.

We propose three different performance metrics to study the performance of our proposal. The first one is the value of the studied attribute (average of the attribute value retrieved from the node chosen for delivery). The bigger this value will be, the better, since we have chosen the maximum relative delivery function. The second indicator is the latency delivery time (time it takes on average for a message to arrive at its destination). Finally, the third indicator is the delivery ratio (average of the number of messages successfully received divided by the total number of messages sent).

\subsection{Simulation Environment and Settings}

In this experimentation, for both scenarios, we simulate a group of different nodes that form a communication network similar in attributes as the one proposed in [3], a study of an intermittently connected emergency scenario. In the two different areas presented in the previous section, different nodes with six different node target profiles share the network. The number of nodes per profile is 50 . Buffer size for the network nodes is fixed to 500MB, which is a realistic value if we consider current mobile phones. Nodes carry an IEEE 802.11g wireless interface controller operating in Ad Hoc mode with a transmit speed fixed to $54 \mathrm{Mbs}$. Maximum range was set to $150 \mathrm{~m}$. Relcast messages created during the simulations have a fixed size of 100KB. Messages are created periodically using a random value between 1 and 10 seconds. The total number of created Relcast messages is 100. Messages are routed

${ }^{7} \sigma=P_{s} / e$ for the maximum relative delivery function, as described in Section 3.4.1 


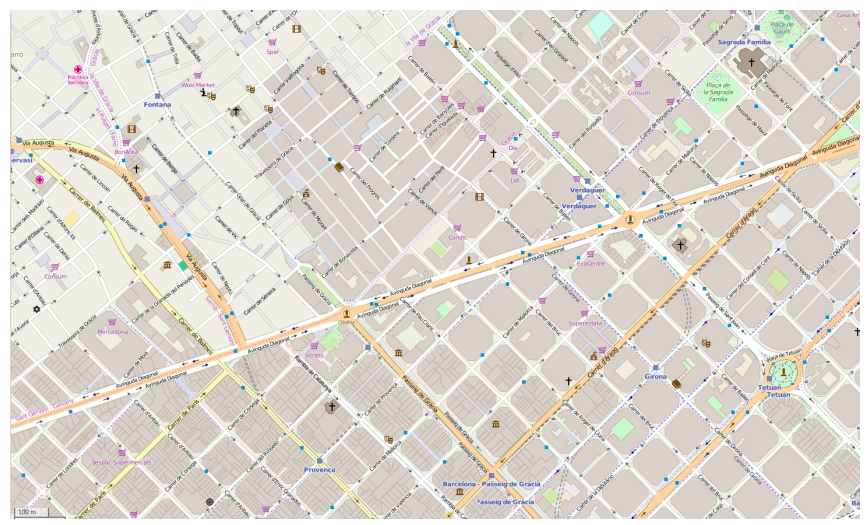

(a) Urban scenario: Eixample area in Barcelona.

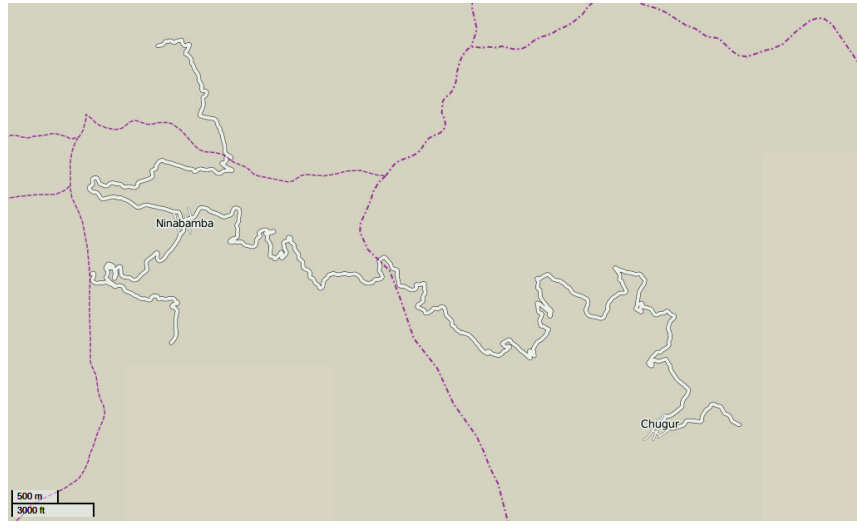

(b) Rural scenario: two towns in the Cajamarca area.

Figure 5: Representation of the two scenarios studied during the experimentation.

using a single-copy routing protocol. Every simulation is repeated 50 times with different random seeds. These random seeds define the random component of the node's movement model.

The experimentation in this section has been conducted using an extension of the Opportunistic Network Environment (The ONE) simulator [18]. This simulator was originally created to run simulation experiments on OppNet and to provide reports and graphical representations of the results. The ONE represents the communication among network nodes and their movement. We have extended The $\mathrm{ONE}^{8}$ to simulate our proposal presented in Section 3.

In Table 2, a description of all the variables and their values used in the simulations is presented.

\subsection{Active-DTN Settings}

For the sake of simulation accuracy, we have performed the simulations presented in this sections following the behaviour and performance of a real Bundle Protocol implementation capable of reproducing the Explore and Wait delivery scheme. We base our simulations on Active-DTN [3], a DTN solution ${ }^{9}$ that consists in extending the Bundle Protocol messages being communicated by incorporating software and message state for forwarding, delivery, lifetime control and prioritisation purposes.

Active-DTN uses RFC 6258 [30], an Bundle Protocol extension block that defines how a bundle can be extended by means of adding a set of Metadata Extension Blocks (MEB). The purpose of MEBs, as defined in [30], is to carry additional information that nodes can use to make processing decisions regarding bundles. Active-DTN defines a Mobile code Metadata Extension Block (MMEB)

\footnotetext{
${ }^{8}$ The source code for this The ONE extension can be found at https://senda.uab.cat/wiki/aDTN in Section "The ONE resources".

${ }^{9}$ Active-DTN source code can be found at https://github.com/SeNDA-UAB/aDTNPlus.
}

\begin{tabular}{|l|c|}
\hline Variables & Value \\
\hline \hline Scenario Size (rural) & $24 \mathrm{~km}^{2}$ \\
\hline Scenario Size (urban) & $8 \mathrm{~km}^{2}$ \\
\hline Transmission Speed & $1 \mathrm{MB} / \mathrm{s}$ \\
\hline Transmit Range & $100 \mathrm{~m}$ \\
\hline \# Nodes & 200 \\
\hline \# Nodes target profile & 50 \\
\hline \# Messages target profile & 100 \\
\hline Max Node Speed & $1.4 \mathrm{~m} / \mathrm{s}$ \\
\hline$\#$ random seeds & 50 \\
\hline Buffer Size & $500 \mathrm{MB}$ \\
\hline Delivery Max Time & $3 \mathrm{~h}$ \\
\hline$\alpha$ (urban) & 0.25 \\
\hline$\omega$ (urban) & 0.95 \\
\hline$\gamma$ (urban) & 0.5 \\
\hline$\delta$ (urban) & 0.1 \\
\hline$\alpha$ (rural) & 0.85 \\
\hline$\omega$ (rural) & 0.45 \\
\hline$\gamma$ (rural) & 0.9 \\
\hline$\delta$ (rural) & 0.9 \\
\hline Message Payload & $300 \mathrm{bytes}$ \\
\hline Mobile Code Extension Size & $54884 \mathrm{~ns}$ \\
\hline Compilation Time & ns \\
\hline Delivery Execution Time & 360 \\
\hline
\end{tabular}

Table 2: Simulation settings default values for both scenarios. 
Node density as a function of the position.

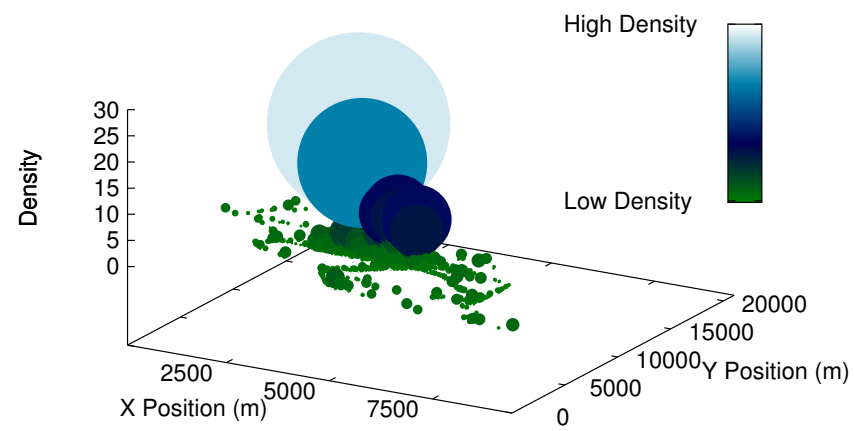

Node density as a function of the position.

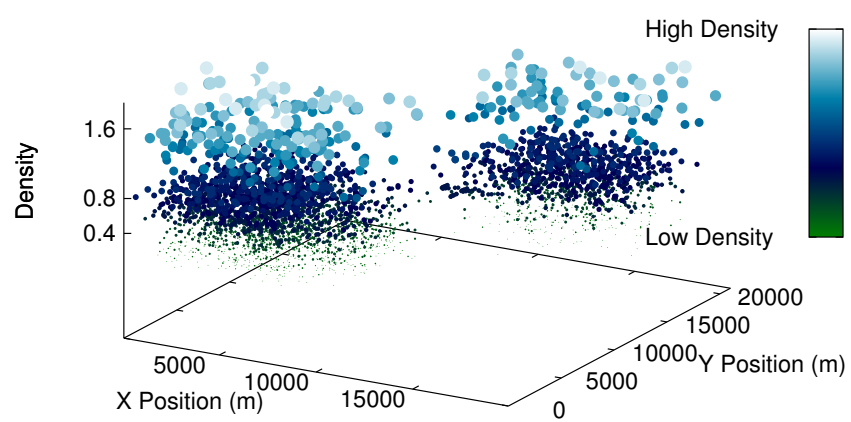

(a) Urban scenario node density: Points of interests are very dense. (b) Rural scenario node density: Density is equally sparse all over the two towns while the road that connects both towns remains less dense.

Figure 6: Study of the urban and rural scenario node density.

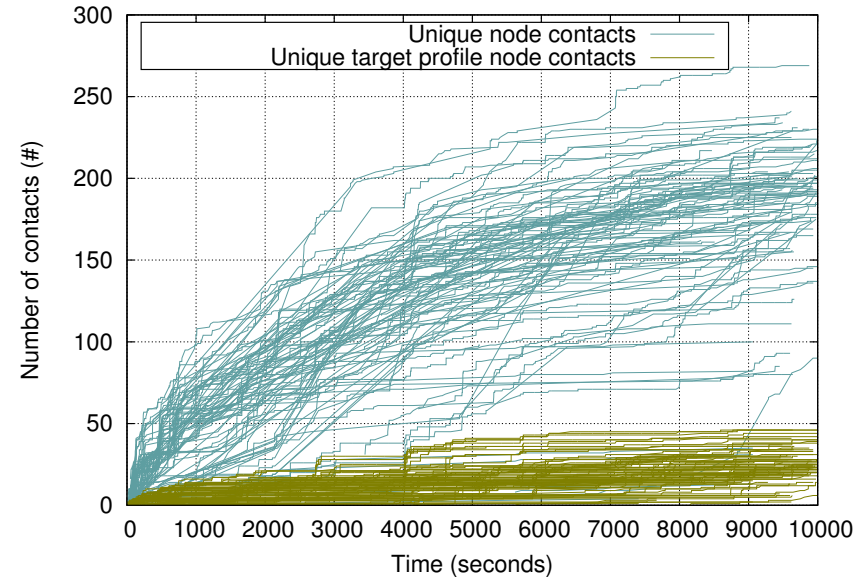

(a) Message Profile Scope in urban scenario.

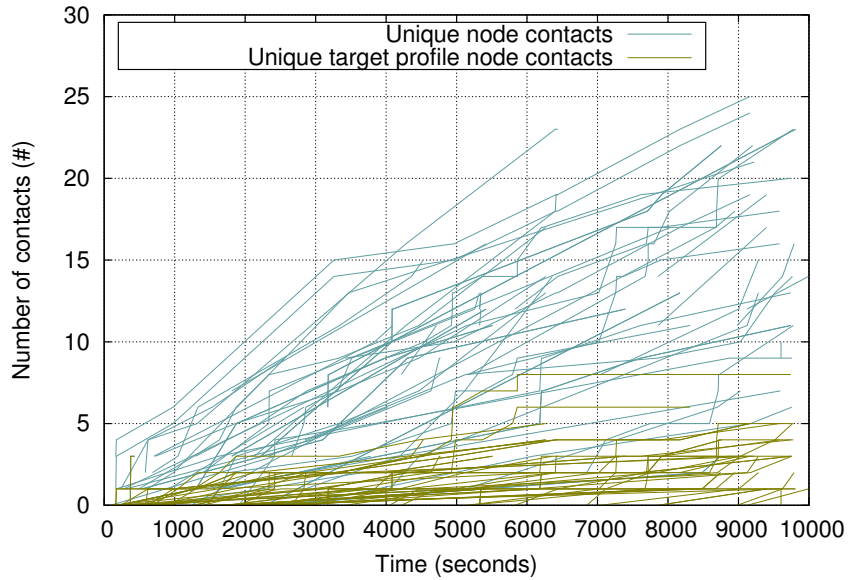

(b) Message Profile Scope in rural scenario.

Figure 7: Study of the Message Profile Scope for the urban and rural scenario. 
as a type of MEB with the necessary fields for the inclusion of software code for routing purposes.

In the following simulations, the routing-delivery strategies presented in Section 3 are included in DTN messages as bundle forwarding and/or delivery extensions following the bundle extension introduced in [3] that is compatible with RFC 6258 [30]. We simulate Relcast messages, ( $m$ (source, payload, p, relf $)$ ), as messages that carry a delivery extension with a software mobile code that implements the relative delivery function $($ relf $)$. The simulator is programmed to allow messages, when carrying the delivery extensions, to be delivered in terms of mobile software codes that are included in these delivery extensions.

As introduced in Section 3, there are several variables that define the state of a message: the Smooth Frequency time (SFt), $\alpha$ (the exponentially weighted moving average constant), for example. These state variables and constants must be kept when being forwarded from one DTN node to another and represent historical information that allows the Explore and Wait scheme to be capable of performing appropriately upon arriving at new nodes. Additionally, this state can be very useful to communicate among forwarding and delivery software codes. For these purposes, we have modified the simulator to extend its messages to allow permanent state data storage and intersoftware codes communication.

In Table 2, details related to Active-DTN considered for the simulations such as the mobile code extension size, delivery mobile code compilation and execution time are described.

\subsection{Scenario Models}

In Figure 5(a) and Figure 5(b), a graphical representation of both scenarios is depicted. As it can be seen in Figure 6(a) and Figure 6(b), nodes in both areas behave in different ways in terms of movement models. In the urban scenario, nodes are tighter coupled to points of interests than in the rural area, where nodes are more spread over the two villages of Ninabamba and Chugur.

In Figure 7(a) and Figure 7(b), we analyse the observed Message Profile Scope $\left(P_{s}\right)$, as defined in 3.6, when 100 Relcast messages are sent to the network for both studied scenarios. These special Relcast messages behave in such a way that they always remain in the Explore phase. In order to understand the difference between both scenarios, we analyse how many different nodes in total and belonging to the target profile theses messages can be forwarded to in a period of 3 hours. As it can be seen, in the urban scenario, there are some messages that are able to be forwarded to every node in the network belonging to the target profile. Instead, in the rural scenario, messages are being forwarded, on average, to a $10 \%$ of the nodes belonging to the target profile.

As a result of these experiments, we see that these two scenarios are different in terms of network topology, node's movement models and the number of total and target pro-

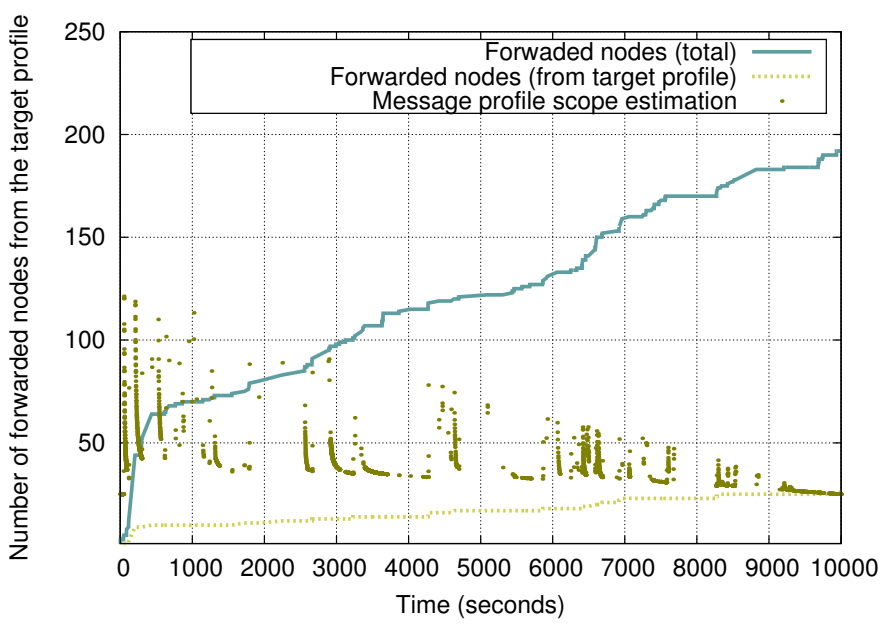

Figure 8: Example of the evolution of the estimation of the Message Profile Scope $\left(P_{s}\right)$. During the early stage of the simulation, the value for this estimation is significantly large in comparison to the real contacted nodes. As the simulation evolves, this estimation gets more realistic. The Relcast message is delivered after switching to the Wait phase, when the message has been forwarded to $\sigma=P_{s} / e$ nodes. In this example, value for $\alpha$ was 0.5 .

file nodes being forwarded to per message. In the following, sections we discuss how to apply the Explore and Wait scheme for both scenarios.

\subsubsection{Explore Phase Experimentation}

We have analysed the estimation of the Message Profile Scope, the average of the estimation of the number of potential nodes belonging to a certain target profile for 100 Relcast messages for a period of 3 hours. We study how $\alpha$ and $\omega$ affect to this Message Profile Scope estimation calculation. As introduced in Section 3, this $\alpha$ and $\omega$ are used to calculate the SIFT and the Measured Profile Membership moving averages respectively.

To understand how this estimation evolves, we represent in Figure 8, as an example based on the urban scenario, the estimation of a Relcast message $P_{s}$ as a function of the simulation time. As it can be seen, this estimation evolves in an irregular way, and, as the simulation evolves it is corrected using the moving averages presented in Section 3.6 .

Additionally, in Figure 9(a) and Figure 9(b), we analyse the Message Profile Scope estimation error percentage for the two different scenarios for 100 Relcast messages sent. During these simulations, we program the simulator to avoid message deliver so we can understand the estimation error. We compare the value of the Message Profile Scope at the moment where the messages have been forwarded to $\sigma$ (optimal Phase Transition Value) nodes with the observed number of nodes a message is forwarded to after 3 hours of time.

The error percentage is calculated by the following expression: 


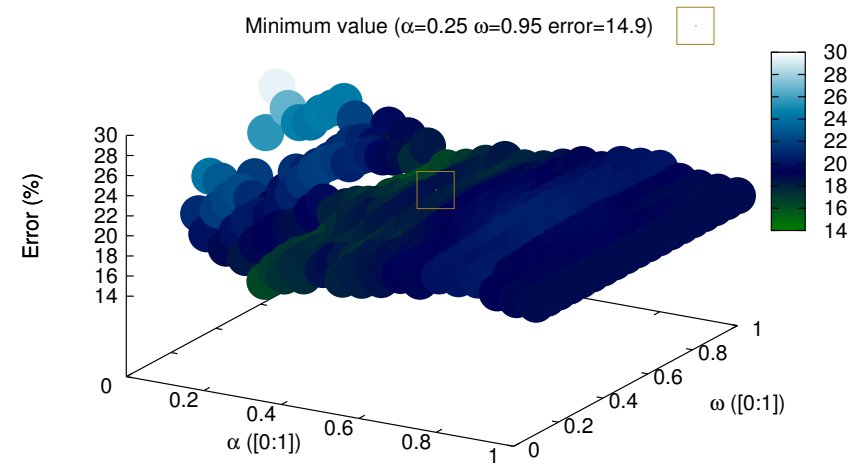

(a) Study of the estimation of the Message Profile Scope for 100 Relcast messages as a function of $\alpha$ and $\omega$. Urban scenario.

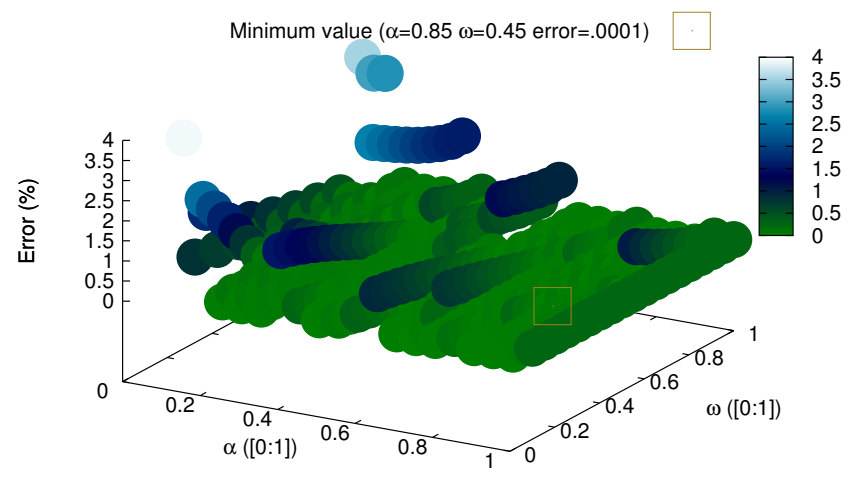

(b) Study of the estimation of the Message Profile Scope for 100 Relcast messages as a function of $\alpha$ and $\omega$. Rural scenario.

Figure 9: Study of the estimation of the Message Profile Scope for 100 Relcast messages as a function of $\alpha$ and $\omega$.

$$
\text { perror }=P_{s(\sigma)} / P_{s(\text { observed })} * 100
$$

where $P_{s(\sigma)}$ is the estimation of the Message Profile Scope at the moment when the message has been forwarded to $\sigma$ (optimal Phase Transition Value) nodes and $P_{s \text { (observed) }}$ the observed number of nodes a message is forwarded to after 3 hours of time.

As it can be seen, these errors are different for the two scenarios. In the urban scenario the smallest error is obtained for small values of $\alpha$ and high values of $\omega$, while, in the rural scenario, the minimum errors are obtained with high values of $\alpha$ and medium values of $\omega$. The reason that for the same $\alpha$ values for the two scenarios we obtain opposite performances is related to the differences on the density of the scenario. In the urban scenario, as depicted in Figure 6(a), the density of groups of users is unstable, while in the rural scenario, as depicted in Figure 6(b), this density is much more stable. For both scenarios, for these optimal values of $\alpha$ and $\omega$ the percentage of error for the estimation of Message Profile Scope is very small $(<15 \%)$. As we will see in the following simulations, this small error allows obtaining performant values of the optimal Phase Transition Value.

\subsubsection{Routing Experimentation}

In the following simulations, the probabilistic profileaware routing protocol, presented in Section 3.3.1, is analysed to understand how it can improve the number of nodes belonging to a target profile a message may be forwarded to during the Explore phase. We compare it with two different routing protocols. The first one is another single-copy routing protocol, the First Contact routing protocol [9], where the message is routed to the first encountered node. Additionally, we compare it with another profile-aware routing protocol, the CSI routing protocol
[14]. As described in Section 2, this protocol makes routing decisions in terms of the mobility profiles of the nodes. CSI works in two phases. In the first phase, the gradient ascent phase, the message explores the network by forwarding a message in a single-copy way until a node with a large similarity with the target profile is found. During the second phase, the group spread phase, the message is replicated and delivered to nodes belonging to the target profile.

In this routing experimentation, we want to compare the gradient ascent phase of the CSI routing protocol with our probabilistic profile-aware routing protocol to understand which of the two routing protocols is better to explore the network in terms of how many nodes belonging to a target profile a message may be forwarded to. We compare the paradigm of studying the probability of contacting nodes from the target profile in a future, as our probabilistic profile-aware routing protocol does, with the one that forwards messages to nodes with high similarity to the target profile higher than the custodian of the message, as in the gradient ascent phase from the CSI protocol.

The experiment compares the behaviour of 100 Relcast messages that remain in the Explore phase and are routed using three different routing protocols: our probabilistic profile-aware routing protocol, First Contact routing protocol and CSI routing protocol ${ }^{10}$. In Figure 10(a), the total number of nodes belonging to a certain target profile the 100 Relcast messages have been forwarded to is studied, for the three routing protocols. As it can be seen in these experiments, our profile-aware probabilistic routing protocol is able to forward the messages to more nodes from the target profile in both scenarios.

\footnotetext{
${ }^{10}$ The source code for the CSI routing protocol for The ONE simulator can be found at https://www.netlab.tkk.fi/tutkimus/dtn/theone/contrib/ProfileCast_CSIT_routing_algorithm.zip
} 


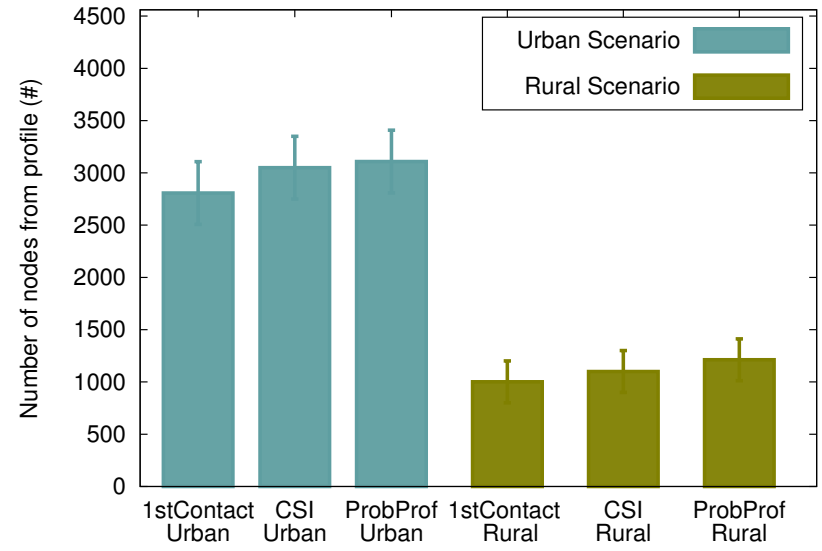

(a) Study of the number of contacted nodes belonging to a certain target profile when 100 Relcast messages are sent to both scenarios for the three routing protocols: First Contact (1stContact), CSI routing protocol (CSI) and our Probabilistic Profile-aware routing protocol (ProbProf).

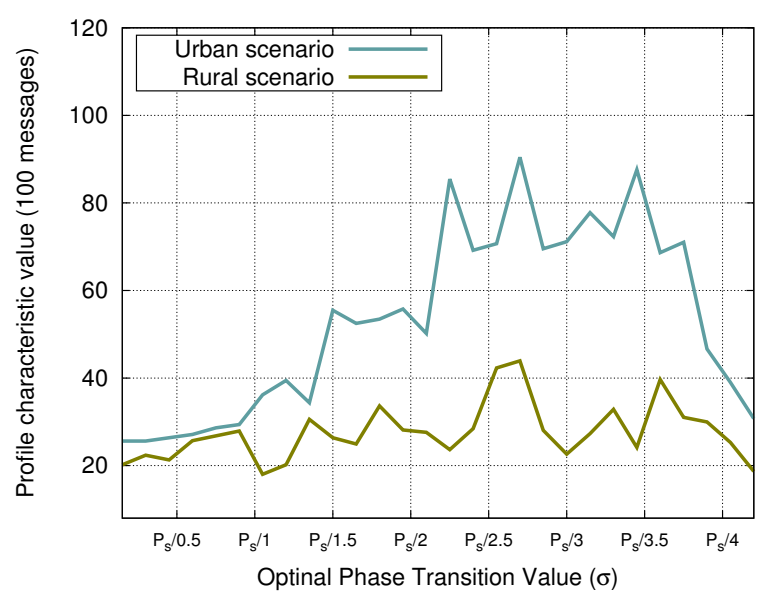

(b) Profile characteristic value obtained by 100 messages as a function of the optimal Phase Transition Value $(\sigma)$.

Figure 10: Routing experimentation: number of contacted nodes belonging to a certain target profile and profile characteristic value obtained.

In Figures 11(a) and 11(b), we analyse the optimal values for $\delta$ and $\gamma$ for our probabilistic profile-aware routing protocol in the two studied scenarios. As it can be seen, low values for $\delta$ for the urban scenario maximise the attribute value obtained, while, for the rural scenario, high values are better. In the very same way, middle values for $\gamma$ in the urban scenario maximise the attribute value obtained while, for the rural scenario, high $\gamma$ obtain the maximum values.

In Figure 10(b), we evaluate how the optimal Phase Transition Value $(\sigma)$ affects the Profile characteristic value obtained when 100 Relcast messages are sent in the two different scenarios. As it can be seen, in both scenarios, maximum Profile characteristic values are obtained when $\sigma$ values are close to $P_{s} / e$.

\subsubsection{Performance Delivery Evaluation}

With all these simulations we have presented in this section, we are now ready to make a general performance evaluation. We make a comparison for three different schemes: a three-phase BroadcastQuery-Response-Unicast scheme, as presented in Section 3.1, an Explore and Wait scheme using both a probabilistic profile-aware routing protocol and a CSI routing protocol. We have sent 100 messages in both studied scenarios using the three schemes. In Figure 12(b), Figure 13(a), Figure 12(a) and Figure 13(b), we compare the three schemes for both scenarios. As it can be seen from these experiments, the Explore and Wait scheme using the probabilistic profile-aware routing protocol performs better than the other two in terms of the total value of the profile attribute obtained from the delivered messages, the delivery ratio, the network overhead and the latency time.

\section{Conclusions}

In this paper, we have presented Relcast, a relative Profile-cast model for OppNet. This model is designed for OppNet scenarios where users would want to send messages to relative profiles defined by relative delivery functions: nodes belong to these relative profiles taking into account not only attributes from the very same node, but also relative to others from the same profile.

We have presented Explore and Wait, a novel composite routing-delivery scheme to improve OppNet Relcast Profile-casting. Explore and Wait operates following two phases: an explore phase, where Relcast messages study the network and understand about the nodes attributes and a delivery phase, where Relcast messages have acquired enough knowledge about the network, and they are ready to be delivered. We have seen that optimal stopping theory are extremely helpful to decide when to perform the phase transition.

Additionally, in order to improve the number of contacted nodes from a certain profile during the explore phase, we have proposed a profile-based probabilistic routing protocol: messages are routed in terms of historical profile encounters.

We have shown, using simulations from two different scenarios, that Explore and Wait routing-delivery scheme performs better than traditional approaches that use state-ofthe-art routing-delivery primitives. The Explore and Wait scheme allows implementing complex delivery strategies that are not able to be achieved using traditional network primitives. Consequently, new applications, such as the ones introduced in this article, can emerge in very different OppNet scenarios. 


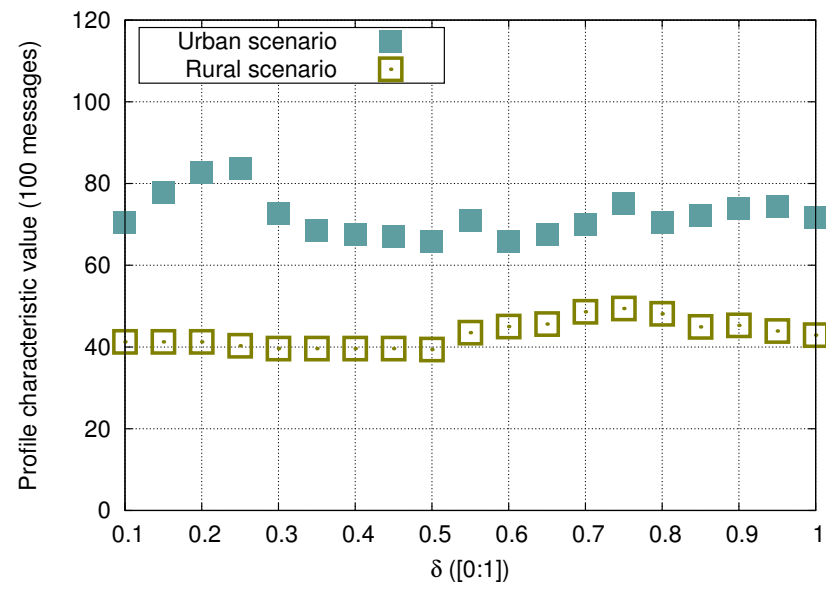

(a) Study of the summation of the attribute value obtained from 100 Relcast messages in 3 hours as a function of the transitivity variable $(\delta)$ using our probabilistic profile-aware routing protocol for the urban and the rural scenario.

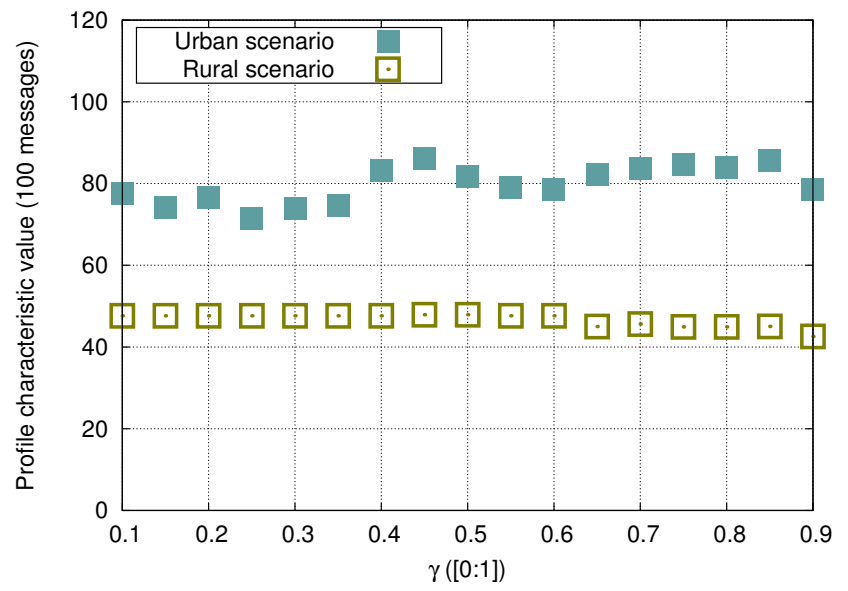

(b) Study of the summation of the attribute value obtained from 100 Relcast messages in 3 hours as a function of the ageing variable $(\gamma)$ using our probabilistic profile-aware routing protocol for the urban and the rural scenario.

Figure 11: Study of the summation of the attribute value obtained from 100 Relcast messages in 3 hours as a function of $\delta$ and $\gamma$ using our probabilistic profile-aware routing protocol for the urban and the rural scenario..

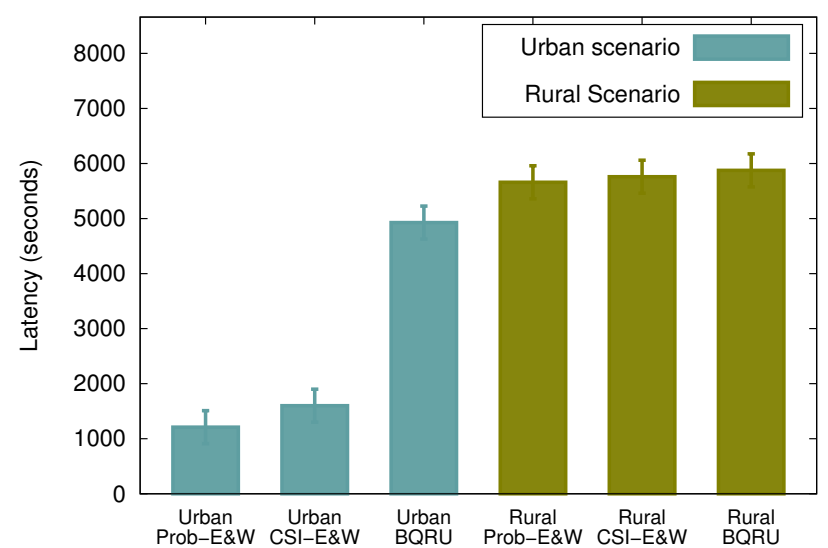

(a) Latency average for three different schemes: Explore and Wait using our Probabilistic Profile-aware routing protocol (Prob-E\&W), Explore and Wait using CSI routing protocol (CSI-E\&W) and BroadcastQuery-Response-Unicast (BQRU).

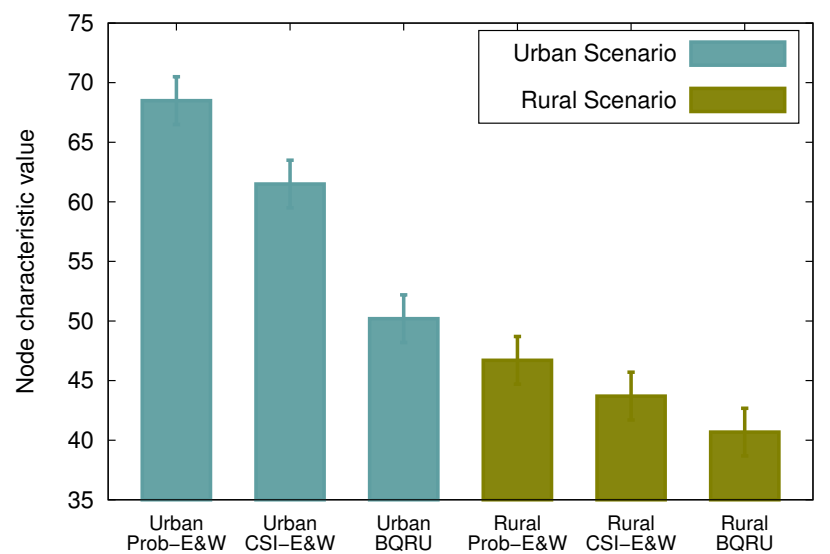

(b) Study of the summation of the attribute value obtained from 100 Relcast messages in 3 hours for three different schemes: Explore and Wait using our Probabilistic Profile-aware routing protocol (ProbE\&W), Explore and Wait using CSI routing protocol (CSI-E\&W) and BroadcastQuery-Response-Unicast (BQRU).

Figure 12: Study of the latency and the attribute value obtained performance. 


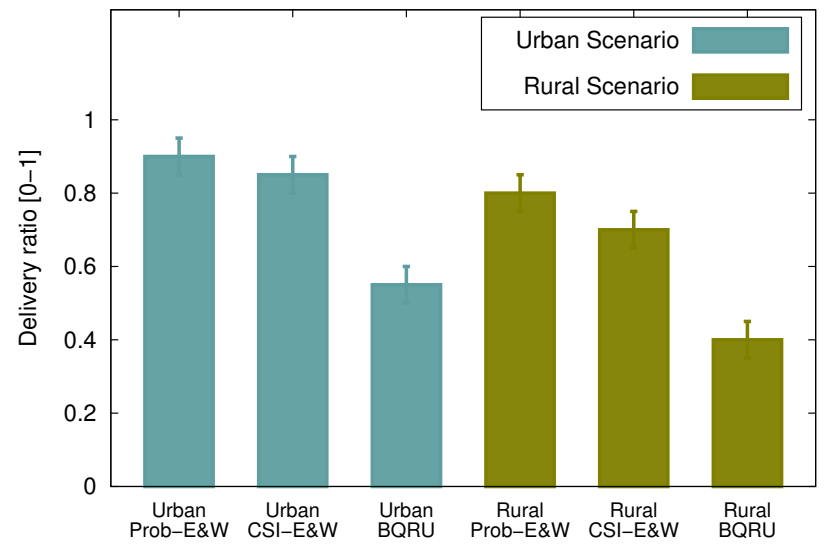

(a) Study of the delivery ratio of 100 Relcast messages in 3 hours for three different schemes: Explore and Wait using our Probabilistic Profile-aware routing protocol (Prob-E\&W), Explore and Wait using CSI routing protocol (CSI-E\&W) and BroadcastQuery-Response-Unicast (BQRU).

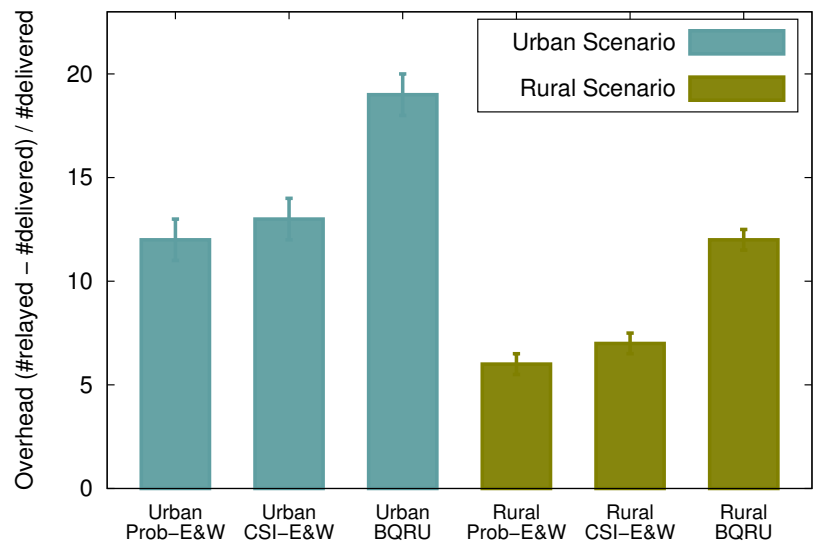

(b) Study of the network overhead ratio average of 100 Relcast messages in 3 hours for three different schemes: Explore and Wait using our Probabilistic Profile-aware routing protocol (Prob-E\&W), Explore and Wait using CSI routing protocol (CSI-E\&W) and BroadcastQuery-Response-Unicast (BQRU).

Figure 13: Study of the delivery ratio and network overhead performance.

\section{Acknowledgements}

This work was partly supported by the Spanish Ministry of Science and Innovation TIN2014-55243-P.

\section{References}

[1] Oladayo Bello and Sherali Zeadally. Intelligent device-to-device communication in the internet of things. IEEE Systems Journal, 10(3):1172-1182, 2016.

[2] Carlos Borrego, Gerard Garcia, and Sergi Robles. Softwarecast: a code-based delivery manycast scheme in heterogeneous and opportunistic ad hoc networks. Ad Hoc Networks, 2016.

[3] Carlos Borrego, Sergi Robles, Angela Fabregues, and Adrián Sánchez-Carmona. A mobile code bundle extension for application-defined routing in delay and disruption tolerant networking. Computer Networks, 87:59-77, 2015.

[4] Ray-Guang Cheng, Nien-Sheng Chen, Yu-Feng Chou, and Zdenek Becvar. Offloading multiple mobile data contents through opportunistic device-to-device communications. Wireless Personal Communications, 84(3):1963-1979, 2015.

[5] Yuan Shih Chow, Herbert Robbins, and David Siegmund. Great expectations: The theory of optimal stopping. Houghton Mifflin Boston, 1971.

[6] Marco Conti, Chiara Boldrini, Salil S Kanhere, Enzo Mingozzi, Elena Pagani, Pedro M Ruiz, and Mohamed Younis. From manet to people-centric networking: milestones and open research challenges. Computer Communications, 71:1-21, 2015.

[7] Elizabeth M Daly and Mads Haahr. Social network analysis for information flow in disconnected delay-tolerant manets. IEEE Transactions on Mobile Computing, 8(5):606-621, 2009.

[8] Xia Deng, Le Chang, Jun Tao, Jianping Pan, and Jianxin Wang. Social profile-based multicast routing scheme for delay-tolerant networks. In Communications (ICC), 2013 IEEE International Conference on, pages 1857-1861. IEEE, 2013.

[9] Mieso K Denko. Mobile Opportunistic Networks: Architectures, Protocols and Applications. CRC Press, 2016.

[10] Daquan Feng, Lu Lu, Yi Yuan-Wu, Geoffrey Ye Li, Gang Feng, and Shaoqian Li. Device-to-device communications underlaying cellular networks. IEEE Transactions on Communications, 61(8):3541-3551, 2013.
[11] Thomas S Ferguson. Who solved the secretary problem? Statistical science, pages 282-289, 1989.

[12] PR Freeman. The secretary problem and its extensions: A review. International Statistical Review/Revue Internationale de Statistique, pages 189-206, 1983.

[13] Wei-jen Hsu, Debojyoti Dutta, and Ahmed Helmy. Profile-cast: Behavior-aware mobile networking. In Wireless Communications and Networking Conference, 2008. WCNC 2008. IEEE, pages 3033-3038. IEEE, 2008.

[14] Wei-jen Hsu, Debojyoti Dutta, and Ahmed Helmy. CSI: A paradigm for behavior-oriented profile-cast services in mobile networks. Ad Hoc Networks, 10(8):1586-1602, 2012.

[15] Pan Hui and Jon Crowcroft. How small labels create big improvements. In Pervasive Computing and Communications Workshops, 2007. PerCom Workshops' 07. Fifth Annual IEEE International Conference on, pages 65-70. IEEE, 2007.

[16] Pan Hui, Jon Crowcroft, and Eiko Yoneki. Bubble rap: Socialbased forwarding in delay-tolerant networks. IEEE Transactions on Mobile Computing, 10(11):1576-1589, 2011.

[17] Esa Hyytiä, Suzan Bayhan, Jörg Ott, and Jussi Kangasharju. Searching a needle in (linear) opportunistic networks. In Proceedings of the 17th ACM international conference on Modeling, analysis and simulation of wireless and mobile systems, pages 187-196. ACM, 2014.

[18] Ari Keränen, Jörg Ott, and Teemu Kärkkäinen. The ONE simulator for DTN protocol evaluation. In Proceedings of the 2nd international conference on simulation tools and techniques, page 55. ICST (Institute for Computer Sciences, SocialInformatics and Telecommunications Engineering), 2009.

[19] Anders Lindgren, Avri Doria, and Olov Schelén. Probabilistic routing in intermittently connected networks. $A C M$ SIGMOBILE Mobile Computing and Communications Review, $7(3): 19-20,2003$.

[20] James M Lucas and Michael S Saccucci. Exponentially weighted moving average control schemes: properties and enhancements. Technometrics, 32(1):1-12, 1990.

[21] Abderrahmen Mtibaa, Martin May, Christophe Diot, and Mostafa Ammar. Peoplerank: Social opportunistic forwarding. In INFOCOM, 2010 Proceedings IEEE, pages 1-5. IEEE, 2010.

[22] Luciana Pelusi, Andrea Passarella, and Marco Conti. Opportunistic networking: data forwarding in disconnected mobile ad hoc networks. Communications Magazine, IEEE, 44(11):134- 
141, 2006.

[23] Dietmar Pfeifer. Extremal processes, secretary problems and the 1/e law. Journal of applied probability, pages 722-733, 1989.

[24] Adrián Sánchez-Carmona, Sergi Robles, and Carlos Borrego. Privhab: a multiagent secure georouting protocol for podcast distribution on disconnected areas. In Proceedings of the 2015 International Conference on Autonomous Agents and Multiagent Systems, pages 1697-1698. International Foundation for Autonomous Agents and Multiagent Systems, 2015.

[25] Adrián Sánchez-Carmona, Sergi Robles, and Carlos Borrego. Identity-based access control for pro-active message's DTN. Security and Communication Networks, 9(14):2323-2337, 2016.

[26] Adrián Sánchez-Carmona, Sergi Robles, and Carlos Borrego. PrivHab+: A secure geographic routing protocol for DTN. Computer Communications, 78:56-73, 2016

[27] Matthias Schwamborn and Nils Aschenbruck. On modeling and impact of geographic restrictions for human mobility in opportunistic networks. In Modeling, Analysis and Simulation of Computer and Telecommunication Systems (MASCOTS), 2015 IEEE 23rd International Symposium on, pages 178-187. IEEE, 2015.

[28] Thrasyvoulos Spyropoulos, Konstantinos Psounis, and Cauligi S Raghavendra. Spray and wait: an efficient routing scheme for intermittently connected mobile networks. In Proceedings of the 2005 ACM SIGCOMM workshop on Delay-tolerant networking, pages 252-259. ACM, 2005.

[29] Sophie Stalla-Bourdillon, Evangelia Papadaki, and Tim Chown. From porn to cybersecurity passing by copyright: how mass surveillance technologies are gaining legitimacy the case of deep packet inspection technologies. Computer Law $\&$ Security Review, 30(6):670-686, 2014.

[30] Susan Symington. Delay-tolerant networking metadata extension block, 2011.

[31] Okan Türkes, Hans Scholten, and Paul Havinga. Roro-lt: social routing with next-place prediction from self-assessment of spatiotemporal routines. In Ubiquitous Intelligence and Computing, 2013 IEEE 10th International Conference on and 10th International Conference on Autonomic and Trusted Computing (UIC/ATC), pages 201-208. IEEE, 2013.

[32] Robert J Vanderbei. The postdoc variant of the secretary problem. Technical Reviews, Princeton, 1995.

[33] Mingjun Xiao, Jie Wu, and Liusheng Huang. Community-aware opportunistic routing in mobile social networks. IEEE Transactions on Computers, 63(7):1682-1695, 2014.

[34] Quan Yuan, Ionut Cardei, and Jie Wu. Predict and relay: an efficient routing in disruption-tolerant networks. In Proceedings of the tenth ACM international symposium on Mobile ad hoc networking and computing, pages 95-104. ACM, 2009.

[35] Mingliu Zhang and Richard S Wolff. Routing protocols for vehicular ad hoc networks in rural areas. IEEE Communications magazine, 46(11), 2008.

[36] Yang Zhang, Wei Gao, Guohong Cao, Tom La Porta, Bhaskar Krishnamachari, and Arun Iyengar. Social-aware data diffusion in delay tolerant manets. In Handbook of Optimization in Complex Networks, pages 457-481. Springer, 2012. 